\title{
Marcadores morfológicos de prognóstico no mesotelioma maligno: um estudo de 58 casos*
}

\author{
ALEXANDRE BOTTREL MOTTA', GERMÂNIA PINHEIRO², LEILA ANTONÂNGELO' ${ }^{1}$, EDWIN ROGER PARRA', \\ MARIA MARGARIDA MONTEIRO', JOSÉ CARLOS DAS NEVES PEREIRA ${ }^{3}$, TEREZA TAKAGAKI ${ }^{4}$, \\ MARIO TERRA FILHO ${ }^{4}$, SANDRO MARTINS ${ }^{4}$, VERA LUIZA CAPELOZZI ${ }^{1}$
}

\begin{abstract}
RESUMO
Objetivo: Diversos marcadores têm se mostrados promissórios como preditores do diagnóstico e prognóstico do mesotelioma maligno (MM). Método: Mediante estudo morfométrico e inmunomarcação de componentes estromais (calretinina, CEA, Leu-M1 e trombomodulina) e nucleares (p53 e Ki-67), avaliamos a sobrevida após o diagnóstico de 58 pacientes com tumores malignos de pleura. Resultados: 0 padrão histológico típico do mesotelioma maligno foi encontrado em 50 casos e o padrão atípico em 8 casos. Imunohistoquimicamente foram confirmados 40 casos como sendo mesoteliomas, 11 como adenocarcimonas e 7 casos do padrão atípico não puderam ser classificados. A análise multivariavel do Cox demonstrou a coexistência de um maior fator de risco de morte (476.2), nos pacientes com idade avançada, subtipo histológico bifásico e componentes de expressão nuclear. Conclusão: A calretinina foi o marcador inmunohistoquímico (lHQ) mais útil para o diagnóstico do mesotelioma e o CEA para o de adenocarcinoma. A quantificação por IHQ da trombomodulina foi fundamental na diferenciação do mesotelioma quando este foi positivo tanto para calretinina e como para o CEA. A informação prognostica mais valiosa foi a fornecida pela análise rotineira histopatológica do tipo histológico tumoral. Um ponto importante, divisor natural, foi a idade com uma media de 55 anos e 30.5\% de componentes nucleares de marcação lHQ, separando os pacientes em dois grupos: pacientes com uma sobrevivência curta contra pacientes com uma sobrevivência mais longa que a esperada. Assim, a análise histopatológica oferece uma arma poderosa e de elevado potencial para guiar no tratamento adjuvante de quimioterápicos após a retirada cirúrgica do mesotelioma.
\end{abstract}

Descritores: Neoplasias pleurais; Mesotelioma; Marcadores biológicos de tumor; Antígeno carcinoembrionário; Prognóstico

* Trabalho realizado na Universidade de São Paulo - USP - São Paulo (SP) Brasil.

1. Graduando em Medicina, Departamento de Patologia da Faculdade de Medicina da Universidade de São Paulo - USP - São Paulo (SP) Brasil.

2. PhD, Departamento de Doenças Ocupacionais do Hospital Universitário Pedro Ernesto da Universidade do Rio de Janeiro - URJ - Rio de Janeiro (RJ) Brasil.

3. PhD, Departamento de Cirurgia Torácica do Hospital das Clínicas da Universidade de São Paulo - USP - São Paulo (SP) Brasil. 4. PhD, Divisão de Doenças Respiratórias e Departamento de Cirurgia Torácica do Hospital das Clínicas da Universidade de São Paulo - USP - São Paulo (SP) Brasil

Endereço para Correspondência: Vera Luiza Capelozzi. Departamento de Patologia da Faculdade de Medicina, Universidade de São Paulo. Av. Dr. Arnaldo 455 - CEP: 01246-903, São Paulo, SP, Brasil. Tel 5511 3066-7427.

E-mail: vcapelozzi@lim05.fm.usp.br

Este estudo foi financiado pelas seguintes agencies brasileiras: Conselho Nacional de Desenvolvimento Científico e Tecnológico (CNPq); Fundação para o Apoio à Pesquisa do Estado de São Paulo (FAPESP); Laboratórios de Investigação Médica (LIMs) 05 e 03, Hospital das Clínicas, Faculdade de Medicina, Universidade de São Paulo (SP) Brasil.

Recebido para publicação: 9/6/05. Aprovado, após revisão em: 8/11/05. 


\section{INTRODUÇÃO}

As estatísticas internacionais quanto à mortalidade devido ao mesotelioma provavelmente subestimam a sua incidência. Isto acontece pois tais casos podem ser descritos como adenocarcinoma ou como outros tumores malignos envolvendo a pleura e o peritônio. Não havendo a disponibilidade de ensaios imunohistoquímicos ou de microscopia eletrônica, estes casos não são diagnosticados. A determinação destes casos via registros de casos de câncer ou via ensaios histopatológicos pode subestimar a verdadeira taxa de mortalidade.

No Brasil, a mortalidade causada por mesotelioma pleural é desconhecida. Ao contrário da sua extremamente baixa incidência na população geral, a população submetida à exposição ocupacional por fibra de asbesto apresenta uma incidência cem vezes maior. ${ }^{(1)}$

As fibras de asbesto são a causa primária de mesotelioma maligno (MM) e apresentam um risco maior no caso de exposição a amosita e crocidolita do que para a exposição a amianto serpentina. ${ }^{(2)}$ Desde 1940 , no Brasil, o asbesto é explorado comercialmente, com a produção de cerca de 200.000 toneladas/ano de amianto serpentina e cerca de 10.000 trabalhadores são expostos a ele. ${ }^{(3-5)}$ Toda a produção é proveniente de duas minas: a mina de São Felix na Bahia e a de Cana Brava em Goiás. Nos estados de São Paulo e Rio de Janeiro, cerca de 90\% da produção de asbesto tem sido utilizada na indústria de fibra de cimento amianto, incluindo fibras de amianto serpentina e crocidolita importadas. Por estas razões, um aumento na incidência de MM é esperado não só no Brasil, mas em muitos países produtores também, com incidências de pico entre 2020 e 2030,(6-8) tornando assim importante o diagnóstico preciso de MM, tanto para o gerenciamento clínico como para decisões médico-legais. A imunohistoquímica (IHQ) é geralmente considerada a técnica auxiliar mais útil no diagnóstico de diversas ocorrências histopatológicas de MM. Entretanto, não há anticorpos completamente sensiveis ou específicos para mesotelioma e há considerável variação nos métodos de coloração. ${ }^{(9-10)}$ Por causa disto, muitos têm estudado outros marcadores a fim de descobrir aquele que possa ser relacionado à morfologia e ao prognóstico de MM. ${ }^{(11-12)}$ Visto que os mecanismos moleculares, tais como as alterações citogenéticas, são considerados importantes para MM, um grupo de genes de supressão tumoral específicos têm sido priorizados como marcadores tumorais potencialmente úteis. ${ }^{(13-14)}$ Entre estes, o marcador tumoral p53 tem se mostrado promissor. 0 p53 é um gene de supressão tumoral específico encontrado em linhagens de células humanas obtidas de pacientes com MM, ${ }^{(15-17)}$ com a utilização de $1 \mathrm{HQ}$, e é encon- trado entre 44\% e 55\% dos mesoteliomas. ${ }^{(18-19)}$

Com o objetivo de abordar este problema, reportamos os aspectos morfológicos e epidemiológicos de 58 casos de MM com a finalidade de explorar a relação entre estes fatores e o prognóstico de sobrevida e o desfecho.

\section{MÉTODO}

Pacientes e tecidos tumorais: 42 pacientes do Hospital das Clínicas da Universidade de São Paulo e 83 pacientes de hospitais do Rio de Janeiro foram diagnosticados com MM entre 1979 e 2000. Os 125 casos envolviam a pleura e em nenhum caso foi descoberto um outro tumor primário. Tecidos obtidos por biópsia, toracoscopia ou necropsia foram obtidos de 58 destes casos. Todas as amostras haviam sido fixadas em formalina e com inclusão em parafina. As biópsias por agulha continham de três a quatro cilindros de tecido, processadas como um bloco de parafina. As biópsias por toracoscopia consistiam de três a quatro pedaços de tecido, o maior dos quais medindo aproximadamente $10 \mathrm{~mm}$ no diâmetro maior. 0 bloco mais apropriado disponível de cada caso foi selecionado e examinado quanto a sua histologia no Departamento de Patologia da Faculdade de Medicina da Universidade de São Paulo ou no Departamento de Patologia do Hospital Pedro Ernesto (RJ). A reavaliação histológica dos subtipos foi realizada por dois patologistas de acordo com a classificação atual da Organização Mundial de Saúde. Nos casos em que a histologia revelou aspectos incomuns para mesotelioma tentou-se um diagnóstico diferencial. Duas categorias principais de diagnóstico histopatológico resultaram deste procedimento: 1) um padrão histológico típico para mesotelioma e 2) uma apresentação atípica. As dificuldades em se atingir um diagnóstico não foram nunca causadas pela pequena quantidade de tecido tumoral disponível, mas sim devido ao próprio quadro histológico. Na Figura 1 (painéis A a F) e na Figura 2 (painéis $A$ a F) apresentam, respectivamente, exemplos de padrão histológico típico e atípico. Os dados fornecedores de informações quanto à epidemiologia e ao prognóstico foram coletados a partir do histórico dos casos e através de entrevistas. 0 tempo de sobrevida foi calculado a partir da data do diagnóstico. Outros detalhes a respeito dos pacientes selecionados para o estudo estão resumidos na Tabela 1 .

Análise imunohistoquímica tumoral: Foram analisadas a presença de antígeno carcinoembriogênico (CEA), Leu-M1, p53, Ki-67, trombomodulina e calretinina através de coloração $1 \mathrm{HQ}$, utilizando-se as téc- 
nicas de imunoperoxidase com o complexo avidina-biotina-peroxidase, de recuperação antigênica por calor úmido em panela a vapor, $\lg$ anti-rato de coelho biotinilado (diluição, 1:400; Dako A/S, Glostrup, Dinamarca), estreptavidina combinada in vitro com peroxidase de rabanete biotinilada (diluição, 1:1000; Dako A/S), tetrahidrocloreto de diaminobenzidina, e contra coloração com hematoxilina. Os anticorpos utilizados foram: CEA monoclonal clone 11-7 (diluição, 1:400; Novocastra, Newcastle, Inglaterra), Leu-M1 clone C3-01 (diluição, 1:20; Biotest, Dreieich, Alemanha), anticorpo monoclonal de rato antiproteína p53 humana (D0-7; diluição, 1:20; Dako A/S), antígeno Ki-67 (diluição, 1:400; Dako A/S) e anticalretinina clone NCL (diluição 1:200; Chemicon, Temecula, CA, EUA). A coloração nuclear marrom foi considerada como evidência da expressão antigênica das células para p53, Ki-67, calretinina e trombomodulina, ao passo que a coloração das membranas e do citoplasma caracterizou a expressão de CEA e Leu-M1. Nós quantificamos a coloração como descrito abaixo. Inicialmente, com pequeno aumento, selecionamos a região de expressão mais intensa. Em seguida, com aumento de 400x, utilizamos uma ocular contendo um retículo com 100 pontos e 50 linhas para a contagem sistemática do número de eventos (núcleos ou citoplasmas positivos para a coloração) cobrindo as linhas do retículo. Calculamos a media de 10 campos microscópicos para a obtenção de um resultado final definido como uma medida quantitativa das estruturas positivas para a coloração. Exemplos de coloração para CEA, Leu-M1, p53, Ki-67, trombomodulina e calretinina são mostrados nas Figuras 3A - 3F.

Análise morfométrica tumoral: Visto que os mesoteliomas apresentam formas histológicas confusas e heterogêneas, que levam a grandes diferenças entre observadores, utilizamos a técnica de contagem de pontos e o mesmo retículo da ocular utilizado para a imunocoloração para avaliar a proporção de textura tumoral de componentes do núcleo e do estroma por fotometria. ${ }^{(20)}$ Os resultados estão expressos como uma razão de pontos cobrindo núcleos ou estromas em relação ao total de pontos do retículo, calculando-se a média de 10 campos tumorais não coincidentes com aumento microscópico de 400x.

Análise estatística: As associações do padrão histológico (típico ou atípico) com as características do tumor (análise da textura e imunohistoquímica) foram realizadas através do teste $\mathrm{U}$ de Mann-Whitney, e dos testes de Kruskal-Wallis e ANOVA. As aná- lises iniciais foram realizadas utilizando-se as curvas de Kaplan-Meier e as análises multivariadas finais foram feitas através da análise de risco proporcional de Cox. Todos os procedimentos utilizados para a avaliação estatística foram realizados utilizando-se o programa SPSS, versão 11.0 (SPSS Inc., Chicago, IL, EUA). 0 limite de significância estatística foi considerado como $p=0,05$.

\section{RESULTADOS}

Diagnóstico histopatológico: Dos 58 casos, a reavaliação do hematoxilina e eosina (H\&E) caracterizou o padrão histológico típico para mesotelioma em 50 casos e o padrão histológico atípico em 8 casos.

Análise tumoral imunohistoquímica CEA: A média de imunocoloração por CEA foi de 2,1 (faixa, 0-35). 0 padrão de coloração foi em geral difuso, intra-citoplasmático. Dos 58 casos, a imunocoloração por CEA foi detectada em 8 casos $(13,8 \%)$, 5 dos quais foram classificados como padrão histológico típico para mesotelioma no H\&E. Os 50 casos remanescentes foram negativos para a imunocoloração por CEA.

Leu-M1: A imunocoloração por Leu-M1 variou entre 0 e 83,1 (media de 4,8) e foi detectada em 17 $(29,3 \%)$ dos 58 tumores. Quatro casos positivos pertenciam à categoria de padrão histológico atípico para mesotelioma. Dos 17 casos positivos, 8 foram também positivos para CEA e considerados adenocarcinomas. 0 padrão de coloração foi difusamente intra-citoplasmático na maioria das vezes, onde estavam presentes formações sólidas; uma acentuação perinuclear foi algumas vezes observada.

Trombomodulina: Nove $(15,5 \%)$ dos tumores demonstraram predominantemente coloração da membrana (Figura 2F) que era focal ou moderadamente difusa na imunocoloração com trombomodulina (média de 0,6; faixa de 0 a 15,0). Todos os exemplos pertenciam à categoria típica.

Calretinina: Em 27 (46,6\%) dos 58 tumores foi demonstrada a calretinina. A imunocoloração foi nuclear e citoplasmática (Figuras 3D, 3E, 3F) com intensidade variando entre 0 e 72,6 (média de 10,2). Todos os casos positivos pertenciam à categoria de diagnóstico típico.

P53: Vinte e nove (50\%) tumores demonstraram imunocoloração para p53 de moderada a intensa (média de 4,8; variando de 0 a 46,0). Vinte e cinco dos casos positivos foram categorizados como diagnóstico histológico típico para mesotelioma. A Fi- 
gura 3B é um exemplo da imunocoloração por p53.

Ki-67: A imunocoloração por Ki-67 variou entre 0 e 55,9 (media de 7,1 ) e foi expressa em 45 $(77,6 \%)$ dos 58 tumores. Oito casos positivos foram categorizados como de padrão histológico atípico e 37 pertenciam à categoria de diagnóstico típico. 0 padrão de coloração foi difusamente intracitoplasmático na maioria dos casos; onde havia formações sólidas; observou-se algumas vezes uma acentuação perinuclear (Figura 3A).

\section{Subtipos histopatológicos}

Quarenta casos de mesotelioma e 11 de adenocarcinoma foram confirmados por $\mathrm{HHQ}$ mas esta foi incapaz de classificar 7 casos com padrão histológico atípico para mesotelioma. Entre os casos de mesotelioma, 24 foram positivos para calretinina, 9 para trombomodulina, 8 para Leu-M1 e 1 caso para CEA em extremamente pequena quantidade. Classificou-se como adenocarcinoma 5 dos 12 casos classificados como de padrão histológico atípico para mesotelioma e 6 dos 46 casos pertencentes à categoria típica. Entre estes 11 casos de adenocarcinoma, 7 foram positivos para CEA, 8 para Leu-M1 e 3 para calretinina. Após a exclusão dos casos de adenocarcinoma por $1 \mathrm{HQ}$, a determinação dos subtipos entre os casos de mesotelioma revelou 22 do tipo epitelial (Figura 1A, 1B), 12 do tipo bifásico (Figuras 2C, 2D), 5 do tipo sarcomatóide (Figuras 3E, 3F) e 1 do tipo desmoplásico.

\section{Análise morfométrica tumoral}

A Tabela 1 resume as associações entre os diagnósticos histológicos com as características tumorais.

O componente tumoral epitelial foi estimado através da fração nuclear ocupada nas células tumorais. As frações nucleares médias foram semelhantes entre os padrões histológicos típicos (21,7\% $\pm 8,8 \%)$ e atípicos $(21,7 \% \pm 10,5 \%)$ e não atingiram significância estatística $(p=0,30)$. As frações nucleares médias encontradas entre os subtipos histológicos foram: $23,2 \% \pm 9,6 \%$ para epitelial; $19,8 \% \pm 8,7 \%$ para bifásico; $18,2 \% \pm 8,1 \%$ para sarcomatóide; $14,1 \% \pm 0 \%$ para desmoplásico; e $21,6 \% \pm 5,6 \%$ para adenocarcinoma. Esta diferença não foi estatisticamente significativa $(p=0,40)$.

Nenhuma diferença estatística foi observada na proporção do componente estromal nos 58 tumores após a estratificação em padrão histológico típico e atípico $(p=0,90)$. A proporção de tumor estromal em tumores atípicos for de 32,5\% $\pm 16,7 \%$ e
$37,5 \% \pm 17 \%$ para os casos típicos. A proporção estromal média aumentou como segue: epitelial $(34,1 \% \pm 12,7 \%)$, adenocarcinoma $(36,7 \% \pm 16 \%)$, desmoplásico (37,6\% $\pm 0 \%)$, sarcomatóide $(37,7 \%$ $\pm 20,7 \%)$, e bifásico $(45 \% \pm 22 \%)$, mas esta diferença não atingiu significância estatística $(p=0,11)$.

Análise da sobrevida: As taxas de sobrevida individuais variaram de 0 a 100 meses, resultando em uma média de sobrevida de 19,1 meses e mediana de 9,5 meses. As taxas de sobrevida observadas foram: $46 \%$ em um ano; 29\% em dois anos; 20\% em três anos; $11 \%$ em quatro anos e $9 \%$ em cinco anos. A expectativa de vida pareceu ser influenciada por fatores individuais tais como idade, subtipos histológicos e análise da textura tumoral. A variação de p53 e Ki-67, entretanto, não obteve significância estatística. A mediana do tempo de sobrevida dos homens foi de 21 meses, enquanto que das mulheres foi de 16 meses. Para pacientes com menos de 55 anos de idade, a mediana de tempo de sobrevida foi de 12 meses, e para pacientes com mais de 55 anos, a mediana foi de 6 meses. Para os subtipos bifásico, sarcomatóide, desmoplásico e epitelial, a mediana do tempo de sobrevida foi, respectivamente, 6, 1, 1 e 19 meses. A imunocoloração de várias células por p53 indicou uma redução da expectativa de vida (mediana de 6 meses) ao passo que resultados negativos ou com poucas células imunocoradas por este marcador corresponderam a mediana de tempo de sobrevida de 12 meses. Quanto à análise da textura tumoral, o componente nuclear indicou uma redução da expectativa de vida (mediana de 7 meses) enquanto que o componente fibroso resultou em uma mediana de tempo de sobrevida de 8 meses.

Nós também testamos a influência dos fatores individuais na expectativa de vida dentro de um modelo multivariado (Tabela 2). Observamos que, através do controle por idade, subtipos histológicos e análise da textura, os pacientes mais idosos com componente nuclear predominante (Figura 4) apresentavam um fator de risco de morte de 476,2 (intervalo de confiança de 95\%: 17,3 a 13068,3; $p=0,00$ ).

\section{DISCUSSÃO}

Aspectos epidemiológicos: Esta é a primeira tentativa de se apresentar um estudo epidemiológico, morfológico e prognóstico a respeito de mesoteliomas no Brasil, onde a falta de estudos sobre estes tumores dificultam as decisões clínicas e médico-legais. Em São Paulo foi publicado so- 


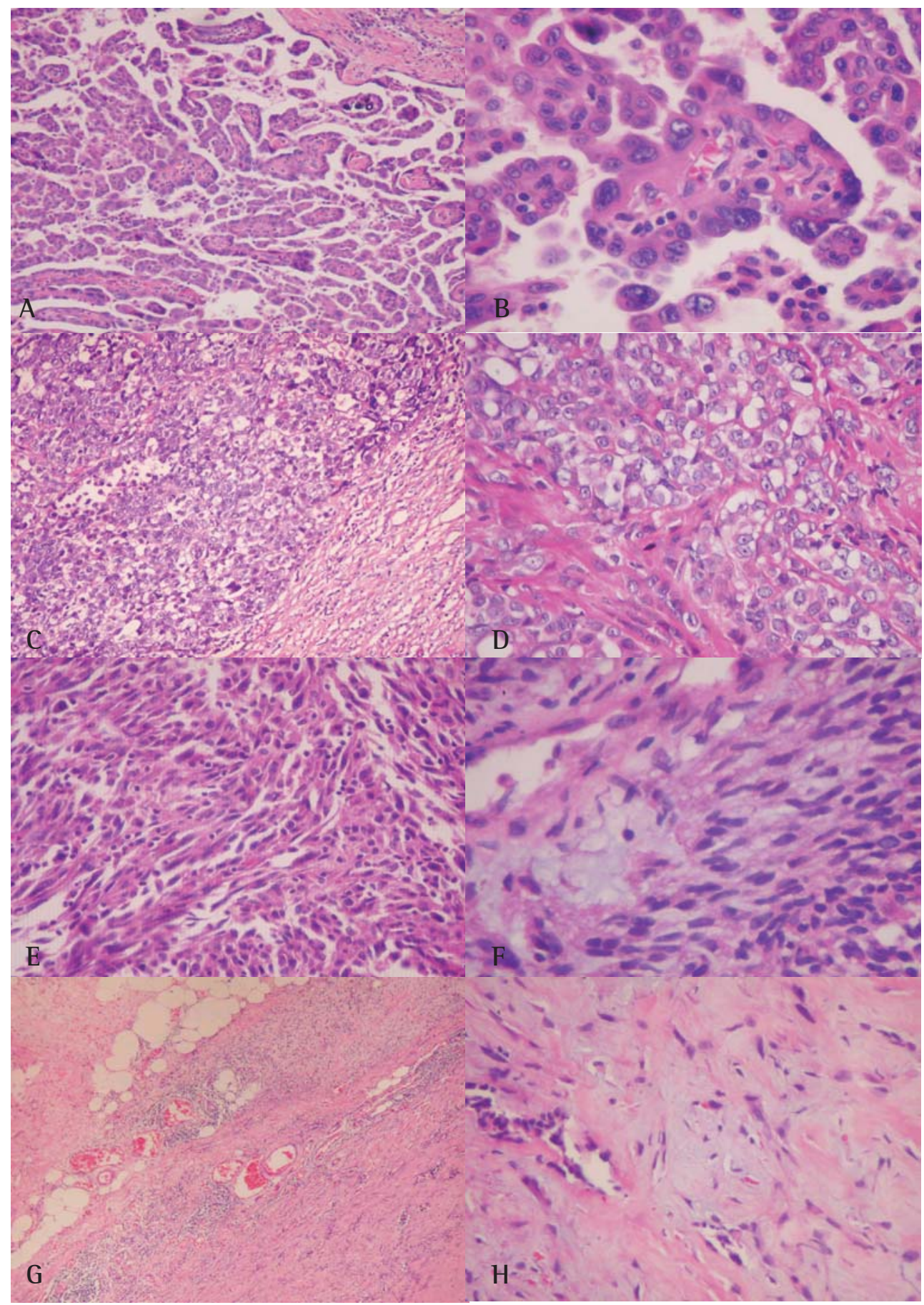

Figura $1(\mathrm{~A}$ a H) - Padrão histológico típico de mesotelioma maligno. Subtipo epitelióide caracterizado por camada de células epiteliais com citoplasma eosinofílico abundante e cromatina nuclear vesicular com nucléolos proeminentes (A,B). Uma combinação de padrões sarcomatóide e epitelióide foi típica para mesotelioma maligno bifásico (C); o componente epitelióide foi representado por camadas de células com citoplasma eosinofílico abundante e cromatina nuclear vesicular nuclear com nucléolos proeminentes (D). Fascículos entrelaçados de células fusiformes compõem a visão panorâmica do mesotelioma sarcomatóide maligno (E); em grande aumento, um estroma mixóide também estava presente (F). Padrão estoriforme de espaços em forma de fenda presentes no mesotelioma desmoplásico maligno (G); muito freqüentemente, este subtipo histológico de mesotelioma maligno exigiu diagnóstico diferencial com pleurisia fibrosa (H). H E A,B,C 40x; D 200x; E 40x; F 400x. H E A,C,E,G 40x; B,D,F 400x 


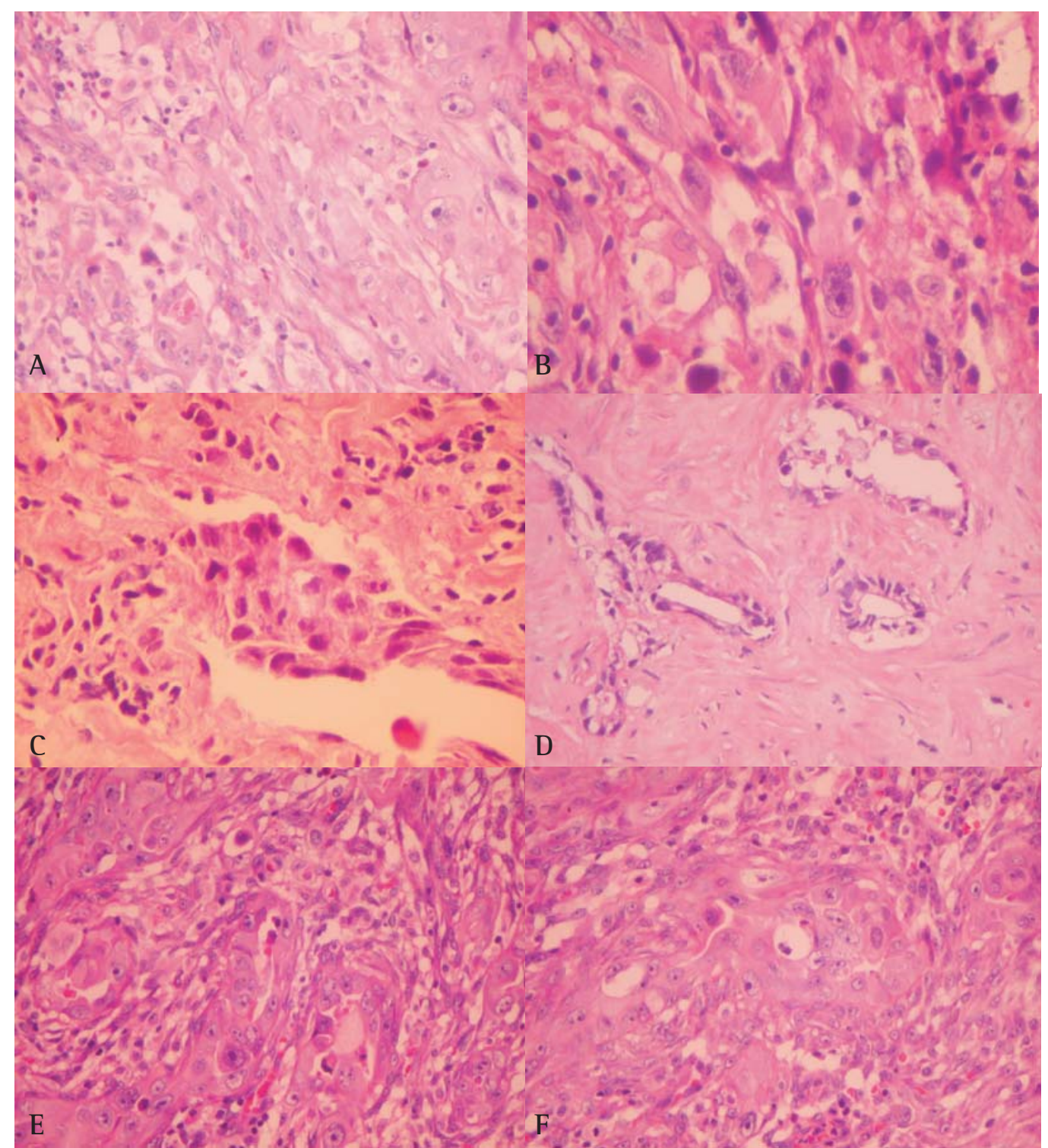

Figura 2 - (A a F) 0 padrão histológico de mesotelioma maligno mostrado nas seções de A a F exige o diagnóstico diferencial entre o subtipo bifásico e o adenocarcinoma com estroma desmoplásico. Este padrão histológico foi considerado atípico para mesotelioma (diagnóstico duvidoso) nas lâminas de $\mathrm{H} \mathrm{E}$, exigindo a complementação com imunocoloração.

e prognóstico para este tipo de tumor, que não pode ser considerado raro em nosso país. A avaliação dos prontuários médicos dos dois Hospitais Universitários não foi um problema para um melhor entendimento dos casos. Entretanto, fomos incapazes de obter dados importantes, tais como a sobrevida, a exposição ocupacional e o estadiamento clínico porque os históricos clínicos estavam incompletos, causando a perda de informações preciosas. Informações a respeito da exposição ao asbesto estavam presentes em somente quatro pron- tuários, mostrando que a história ocupacional não é bem explorada, visto que o asbesto é responsável pela maioria dos casos de mesotelioma. ${ }^{(23)}$ Embora alguns poucos parentes concordaram em cooperar, as entrevistas realizadas com estes não puderam complementar os dados referentes à exposição ao asbesto. Vários fatores podem haver contribuído para esta resposta desapontadora, tais como o baixo nível educacional, mudanças de endereço e o fato de que a entrevista com os familiares não seja um procedimento comum no Brasil. 


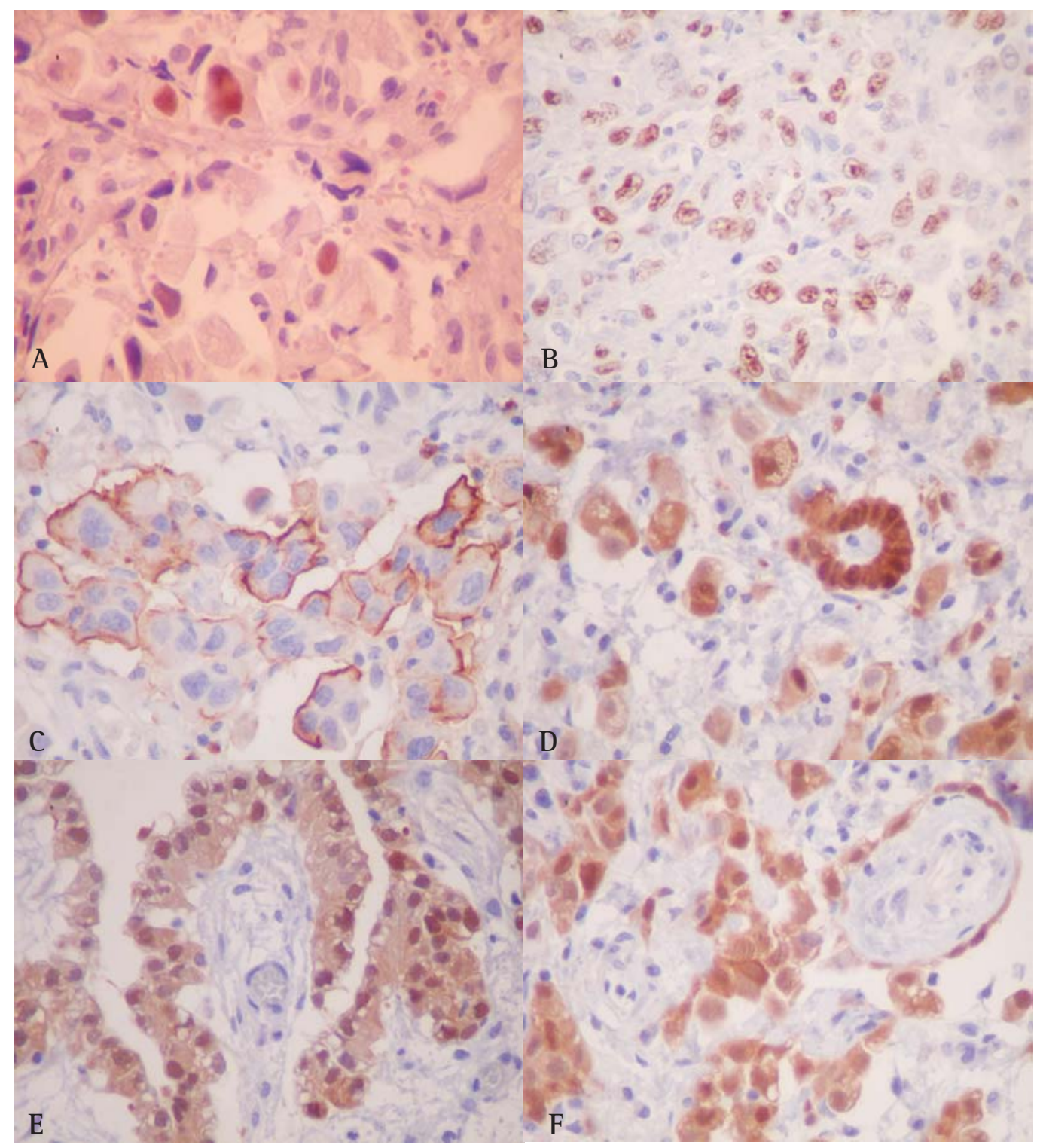

Figura 3 - (A a F) Imunohistoquímica para mesotelioma epitelial maligno: padrão nuclear visualizado para Ki-67 (A) e p53 (B). Note a imunocoloração nuclear homogênea para p53 contrastando com o padrão granular encontrado para Ki-67. Um padrão claramente membranoso foi muito específico para a imunocoloração por trombomodulina, embora algumas células positivas focais estavam presentes (C). Imunocoloração nuclear e citoplasmática para calretinina em padrões histológicos tubular (D) e papilar (E,F) mesotelioma epitelial maligno. Imunocoloração A - F 400x

mente um estudo a respeito de três casos de MM. ${ }^{(21)}$ No Rio de Janeiro, um relatório preliminar sobre o nosso grupo de 83 casos de MM entre 1979-2000 demonstrou que pode haver a subestimação da mortalidade devido a mesotelioma nos atestados de óbito. ${ }^{(22)}$ Entretanto, neste estudo de coorte, além de uma relativamente boa casuística dos mesoteliomas apresentados, tivemos grandes dificuldades em obter um panorama epidemiológico
Aspectos morfológicos: 0s nossos resultados demonstraram que o diagnóstico de MM ainda permanece pouco objetivo mesmo com a experiência a respeito desta malignidade. A revisão histológica mostrou que não é sempre possível chegarse a um diagnóstico confiável somente com base na histopatologia. Até mesmo o subtipo bifásico não pode ser considerado como um critério diagnóstico confiável. A proporção de componentes 


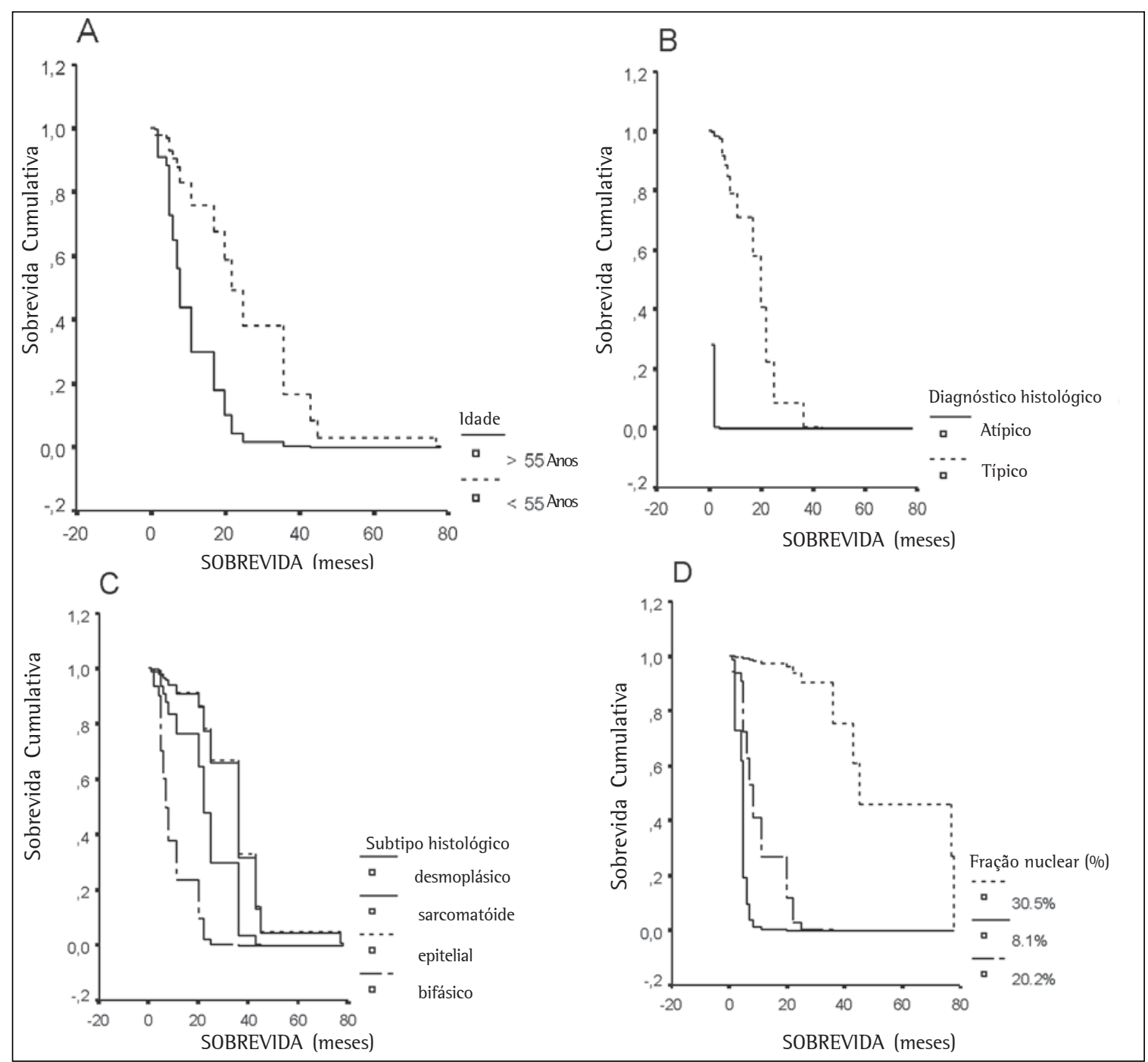

Figura 4 - Gráficos de Cox da probabilidade de sobrevida conta o tempo de seguimento em meses daqueles pacientes com mesotelioma maligno patológico. 0 grupo com = 55 anos de idade (painel A) aparece como a curva esquerda de baixo, e o grupo com < 55 anos de idade aparece como a curva de cima. 0 diagnóstico histológico típico para mesotelioma (painel B) aparece como a curva de cima à direita, e o grupo atípico aparece como a curva de baixo à esquerda. Nos subtipos histológicos (painel C), o mesotelioma maligno bifásico aparece como a curva de baixo. 0 grupo com alta textura epitelial (80\%) aparece como a curva de cima, e o grupo com a proporção de 45 aparece como a curva de baixo.

epiteliais e estromais neste subtipo em nosso estudo, todavia, variou enormemente, provavelmente devido a erros de amostragem que resultaram em dificuldades diagnósticas. Em geral, ao menos 10\% do tumor deve ter um componente fibroso (ou epitelial) para que o MM seja classificado como bifásico. De fato, se fossemos classificar como bifásicos todos os MMs que apresentavam as duas morfologias, a maioria dos mesoteliomas teriam sido classificados como bifásicos. Foram descritos padrões histológicos para o mesotelioma epitelial. ${ }^{(24)}$ Este subtipo é caracterizado por células epiteliais grandes e bem diferenciadas com núcleos em posição central, ausência de atipia e citoplasma abun- dante, que geralmente crescem formando espaços semelhantes a glândulas ou tubulares. Entretanto, alguns mesoteliomas epiteliais crescem formando camadas de células epiteliais. Estes casos típicos foram facilmente reconhecidos como de origem mesotelial neste estudo. Entretanto, estes nem sempre estavam presentes. Variações no quadro histológico, particularmente do mesotelioma epitelial, causaram problemas sérios na obtenção de um diagnóstico. Os adenocarcinomas se pareciam muito com o MM, ou o MM era tão atípico que assemelhava-se a um adenocarcinoma metastático. Nestes casos atípicos, com formações tubulares e tipos de células mais cilíndricas, o adenocarcinoma metastático foi excluído embora não 
TABELA 1

Associações de diagnóstico histológico (típico e atípico) com características tumorais (análise de textura e imunohistoquímica) realizadas através dos testes $\mathrm{U}$ de Mann-Whitney, Kruskal-Wallis e ANOVA. 0 limite de significância estatística foi estabelecido em $\mathrm{p}=0.05$

\begin{tabular}{|c|c|c|c|c|c|c|c|c|c|c|}
\hline \multirow{2}{*}{ Imuno } & \multicolumn{3}{|c|}{ Diagnóstico Histológico } & \multicolumn{7}{|c|}{ Histopathologic subtypes } \\
\hline & Típico & Atípico & & Epitelial & Bifásico & Sarcomat & Desmopl & Adenocarc & Sem Classifica & ão \\
\hline$\overline{\mathrm{CEA}}$ & $5 / 46$ & $3 / 12$ & 0,11 & $1 / 22$ & $0 / 12$ & $0 / 5$ & $0 / 1$ & $7 / 11$ & $0 / 7$ & 0,00 \\
\hline Thromb & $9 / 46$ & $0 / 12$ & 0,10 & $8 / 22$ & $0 / 12$ & $1 / 5$ & $0 / 1$ & $0 / 11$ & $0 / 7$ & 0,11 \\
\hline Calr & $27 / 46$ & $0 / 12$ & 0,03 & $14 / 22$ & $7 / 12$ & $3 / 5$ & $0 / 1$ & $3 / 11$ & $0 / 7$ & 0,30 \\
\hline P53 & $25 / 46$ & $4 / 12$ & 0,26 & $11 / 22$ & $10 / 12$ & $1 / 5$ & $0 / 1$ & $4 / 11$ & $3 / 7$ & 0,36 \\
\hline Nuclear & $21,7 \pm 8,8 \%$ & $21,7 \pm 10,5 \%$ & 0,30 & $23,2 \pm 9,6 \%$ & $19,8 \pm 8,7 \%$ & $18,2 \pm 8,1 \%$ & $14,1 \pm 0 \%$ & $21,6 \pm 5,6 \%$ & $23,9 \pm 13,4$ & 0,40 \\
\hline Estromal & $37,5 \pm 17 \%$ & $32,5 \pm 16,7 \%$ & 0,90 & $34,1 \pm 12,7 \%$ & $45 \pm 22 \%$ & $37,7 \pm 20,7 \%$ & $37,6 \pm 0 \%$ & $36,7 \pm 16 \%$ & $28,2 \pm 17,6$ & 0,11 \\
\hline
\end{tabular}

houvesse uma invasão bruta do pulmão. ${ }^{(25)}$ Para excluir estes símiles, o diagnóstico de MM foi confirmado complementarmente por $\mathrm{IHQ}$.

Neste estudo, demonstramos que os marcadores imunohistoquímicos para mesoteliomas são mais indicativos do que absolutos. Nenhum dos anticorpos utilizados neste estudo foi totalmente sensivel ou específico para o mesotelioma e, de acordo com estudos prévios, houve variação considerável dos padrões de coloração. ${ }^{(9-11)}$ Apesar do uso do painel 1HQ, 7 casos com padrão atípico para mesotelioma foram inseridos na categoria sem classificação devido à falta de expressão associada às formas histológicas confusas e heterogêneas. Entretanto, encontramos um modo quantitativo de reportar o IHQ para marcadores tumorais que pode trazer informações úteis, especialmente nos casos que apresentem aspectos histológicos menos típicos.

Por exemplo, muitos autores demonstraram que a ausência de imunorreatividade ao CEA é característica de mesotelioma, ao passo que muitos outros autores reportaram até $45 \%$ de variação da imunocoloração por CEA.(26) Por esta razão, acreditamos que a positividade ao CEA não deveria excluir o diagnóstico de mesotelioma, mas sim disparar uma investigação abrangente quanto à possibilidade de um outro tumor primário desconhecido. A expressão da imunocoloração por CEA é importante para tumores com aspectos histológicos típicos ou atípicos. Em nosso estudo, 5 casos com padrão histológico típico para mesotelioma expressaram positividade ao CEA (faixa de 1,4 a 35). 0 caso com menor positividade ao CEA também expressou calretinina e trombomodulina em quantidades consideráveis e foi classificado como mesotelioma epitelial. Os outros quarto casos foram re- classificados como adenocarcinoma.

Semelhante ao CEA, os mesoteliomas geralmente não expressam Leu-M1 mas este é expresso em quase todos os casos de adenocarcinoma pulmonar. ${ }^{(27-28)}$ Entretanto, encontramos reatividade fraca e focal (média de 5,4) em 20\% dos mesoteliomas, contrastando com uma média de 28,9 em 73\% dos adenocarcinomas. Os resultados indicam que

\section{TABELA 2}

Análise do modelo de risco proporcional de Cox do tempo de sobrevida

\begin{tabular}{|c|c|c|c|c|c|c|}
\hline \multirow[b]{2}{*}{ Variável } & \multicolumn{6}{|c|}{ 1C95\% para $\operatorname{Exp(B)~}$} \\
\hline & B Coeficiente & e SE & $\mathrm{p}$ & $\operatorname{Exp}(B)$ & Menor & Maior \\
\hline 1dade & 3,8 & 1,30 & 0,00 & 45,6 & 3,3 & 624,5 \\
\hline Sexo & $-0,5$ & 0,70 & 0,26 & 0,6 & 0,2 & 2,2 \\
\hline \multicolumn{7}{|c|}{ Diag. histológico } \\
\hline \multicolumn{7}{|c|}{ Típico } \\
\hline Atípico & $-6,1$ & 3,50 & 0,08 & 0,0 & 2,4 & 2,2 \\
\hline Subtipos & & 0,07 & & & & \\
\hline Bifásico & 3,0 & 2,40 & 0,29 & 20,9 & 0,2 & 2289,6 \\
\hline Sarcom. & 0,1 & 2,10 & 0,94 & 1,2 & 0,0 & 76,3 \\
\hline Desmopl. & 1,3 & 2,80 & 0,63 & 3,7 & 0,0 & 864,1 \\
\hline \multicolumn{7}{|l|}{ Imunohist. } \\
\hline P53 Pontos & & 0,33 & & & & \\
\hline 0 & 0,1 & 1,30 & 0,95 & 1,1 & 0,1 & 13,2 \\
\hline 2,6 & 1,5 & 1,10 & 0,17 & 4,4 & 0,5 & 38,0 \\
\hline K167 Pontos & & 0,51 & & & & \\
\hline 0,3 & $-1,2$ & 1,30 & 0,35 & 0,3 & 0,0 & 3,9 \\
\hline 6,3 & 0,2 & 1,00 & 0,83 & 1,2 & 0,2 & 8,3 \\
\hline \multicolumn{7}{|l|}{ Fraction } \\
\hline Nuclear \% & & 0,00 & & & & \\
\hline 8,1 & 4,4 & 1,70 & 0,01 & 84,2 & 2,8 & 2562,3 \\
\hline 30,5 & 6,2 & 1,70 & 0,00 & 476,2 & 17,4 & 13068,3 \\
\hline Stromal \% & & 0,44 & & & & \\
\hline 19,9 & 3,2 & 2,50 & 0,20 & 23,2 & 0,2 & 3137,0 \\
\hline 55,3 & 1,3 & 1,20 & 0,27 & 3,8 & 0,3 & 43,4 \\
\hline
\end{tabular}

Razão de probabilidade $\log =71,3 ;$ qui-quadrado $=30,9(p=0,01)$ 
a alta expressão de Leu-M1 pode ser útil na diferenciação entre o mesotelioma e o adenocarcinoma.

Recentemente, vários estudos indicaram a importância da calretinina como um marcador positivo para o diagnóstico de mesotelioma, ${ }^{(11,29-30)}$ mas, novamente, uma ampla variação dos padrões de coloração tornam a calretinina um marcador mais indicativo do que absoluto. A valorização da coloração citoplasmática ao invés da nuclear e as diferenças em relação à sensibilidade dos anticorpos são duas das causas implicadas nas discrepâncias entre os resultados. No presente estudo, consideramos a coloração dos núcleos e do citoplasma para calretinina, encontrando positividade em 60\% dos mesoteliomas, com um grande número de células positivas encontradas no subtipo epitelial.

No caso da trombomodulina, a literatura demonstra uma grande discordância na porcentagem de mesoteliomas positivos para a trombomodulina, variando entre $49 \%$ e 100\%. Em nosso estudo, $9 /$ 40 dos mesoteliomas $(22,5 \%)$ reagiram com o anticorpo anti-trombomodulina, indicando uma baixa sensibilidade deste marcador positivo para mesotelioma.

Entre os mesoteliomas com histologia típica e atípica, $56 \%$ e $33 \%$, respectivamente, mostraram coloração nuclear das células tumorais com p53, mas não foram encontradas diferenças entre os subtipos. As discrepâncias nos resultados de diversos estudos podem ser relacionadas à fixação do tecido e à recuperação do antígeno, que afeta fortemente a imunocoloração por p53.

Como em estudos prévios, também vimos que a imunorreatividade ao Ki-67, expressa em 77,6\% dos tumores, não foi útil para o diagnóstico ou ao prognóstico de MM.

Embora os aspectos histológicos e imunohistoquímicos do mesotelioma sejam importantes no procedimento diagnóstico, neste estudo confirmamos que os mesoteliomas levam a grandes diferenças entre observadores. Por este motivo, uma análise da textura, descrevendo os aspectos histológicos do tumor assim como a morfologia das células, foi realizada. Pelo que sabemos, este estudo apresenta a primeira descrição quantitativa da arquitetura do tecido, utilizando parâmetros que descrevem a densidade nuclear e estromal com uma abordagem simples, de rotina e de baixo custo. Entretanto, não nos surpreende que formas histológicas confusas e heterogêneas de mesotelioma tenham sido novamente enfatizadas pela análise de textura, visto que nenhuma diferença significativa foi obtida em termos da fração nuclear ou estromal entre elas. Vale ressaltar que estes achados foram muito importantes na predição do risco de morte dos pacientes com MM.

Aspectos prognósticos: 0 tempo de sobrevida mediano de 9,5 meses está abaixo dos resultados de outras investigações. Um tempo de sobrevida excepcional de 100 meses foi detectado em um paciente cuja descoberta do mesotelioma foi inesperada após uma pleuroctomia devido a pneumotórax espontâneo. Isto pode ser indicativo de que o pneumotórax espontâneo, embora seja um sintoma incomum, possa ser considerado como um sintoma inicial. Somente pacientes assintomáticos ou pacientes com tais sintomas iniciais podem ter um prognóstico melhor. Como em outros estudos, verificamos que o padrão epitelial dos mesoteliomas propicia um prognóstico melhor. Nos padrões bifásico, sarcomatóideo e desmoplásico, o tempo de sobrevida de 6 meses, 1 mês e 1 mês, respectivamente, está de acordo com a literatura, que descreve um pior prognóstico para estes tipos. Claramente, a alta taxa de mortalidade indica que não houve progressos quanto ao diagnóstico durante a fase inicial da doença ou ao tratamento efetivo. A questão de interesse é se as informações adicionais e mais tecnológicas reunidas do tecido tumoral podem nos auxiliar na identificação precoce ou na detecção dos fatores que influenciarão no tratamento. Os nossos resultados sugerem que a análise de textura, controlada pela idade e pelos subtipos histológicos, fornece informações prognósticas mais úteis do que somente a análise histopatológica de rotina. Um divisor natural foi a idade mediana de 55 anos e a presença de $30,5 \%$ de componentes nucleares. Estes pontos forneceram uma forma prática para separar os pacientes em dois grupos: pacientes com uma expectativa de sobrevida curta contra pacientes com expectativa de vida mais longa. Desta forma, a análise de textura nos oferece o potencial para guiar o uso da quimioterapia auxiliar em pacientes que provavelmente não serão beneficiados com a excisão cirúrgica do mesotelioma.

Os nossos resultados indicam que o marcador mais útil para o diagnóstico de mesotelioma foi a calretinina, ao passo que o CEA o foi para o adenocarcinoma. A quantificação $\mathrm{HHQ}$ de trombomodulina auxiliou no diagnóstico de mesotelioma 
quando tanto a calretinina e o CEA eram positivos. A análise de textura forneceu informações prognosticas mais úteis do que a análise histopatológica de rotina, nos oferecendo o potencial na condução do uso de quimioterapia auxiliar em pacientes que provavelmente não seriam beneficiados pela excisão cirúrgica do mesotelioma. São necessários outros estudos envolvendo um número maior de pacientes em um ensaio prospectivo e randomizado para a validação da nossa avaliação quantitativa da análise de textura

\section{REFERÊNCIAS}

1. Walz R, Koch HK. Malignant pleural mesothelioma: some aspects of epidemiology, differential diagnosis and prognosis. Histological and immunohistochemical evaluation and followup of mesotheliomas diagnosed from 1964 to January 1985. Pathol Res Pract. 1990;186(1):124-34.

2. Carbone M, Kratzke RA, Testa JR. The pathogenesis of mesothelioma. Semin Oncol. 2002;29(1):2-17.

3. Costa JLR, Ferreira Jr YM, Mendes R. Asbesto e doença: introdução ao problema no Brasil. Rev Assoc Med Bras. 1983;(1/2):1821.

4. Associação Brasileira do Amianto. 0 amianto no Brasil. 2a ed. São Paulol: Ebart; 1996.

5. Case BW, Dufresne A, Bagatin E, Capelozzi VL. Lung-retained fibre content in Brazilian chrysotile workers. Ann Occup Hyg. 2002;46(1):144-9.

6. Peto J, Hodgson JT, Matthews FE, Jones JR. Continuing increase in mesothelioma mortality in Britain. Lancet. 1995;345(8949):535-9. Comment in: Lancet. 1995;345 (8959):1233. Lancet. 1995;345(8959):1233-4. Lancet. 1995;345(8959):1234.

7. Banaei A, Auvert B, Goldberg M, Gueguen A, Luce D, Goldberg S. Future trends in mortality of French men from mesothelioma. Occup Environ Med. 2000;57(7):488-94.

8. Jarvholm B, Englund A, Albin M. Pleural mesothelioma in Sweden: an analysis of the incidence according to the use of asbestos. Occup Environ Med. 1999;56(2):110-3.

9. Ordonez NG. The immunohistochemical diagnosis of epithelial mesothelioma. Human Pathol. 1999;30(3):313-23.

10. Riera JR, Astengo-Osuna C, Longmate JA, Battifora H. The immunohistochemical diagnostic panel for epithelial mesothelioma: a reevaluation after heat-induced epitope retrieval. Am J Surg Pathol. 1997;21(12):1409-19. Comment in: Am J Surg Pathol. $1997 ; 21(12): 1395-8$.

11. Cury PM, Butcher DN, Fisher C, Corrin B, Nicholson AG. Value of the mesothelium-associated antibodies thrombomodulin, cytokeratin 5/6, calretinin, and $\mathrm{CD} 44 \mathrm{H}$ in distinguishing epithelioid pleural mesothelioma from adenocarcinoma metastatic to the pleura. Mod Pathol. 2000;13(2):107-12.

12. Gordon GJ, Jensen RV, Hsiao LL, Gullans SR, Blumenstock JE, Richards WG, et al. Using gene expression ratios to predict outcome among patients with mesothelioma. J Natl Cancer Inst. 2003;95(8):598-605.

13. Pass HI, Liu Z, Wali A, Bueno R, Land S, Lott D, et al. Gene expression profiles predict survival and progression of pleural mesothelioma. Clin Cancer Res. 2004;10(3):849-59.

14. De Rienzo A, Testa JR. Recent advances in the molecular analysis of human malignant mesothelioma. Clin Ter. 2000;151(6):433-8.
15. Cote RJ, Jhanwar SC, Novick S, Pellicer A. Genetic alterations of the $\mathrm{p} 53$ gene are a feature of malignant mesotheliomas. Cancer Res. 1991;51(19):5410-6. Erratum in: Cancer Res. 1991;51(23 Pt 1):6399. Erratum in: Cancer Res. 1991;51(23 Pt 1):6399.

16. Kafiri G, Thomas DM, Shepherd NA, Krausz T, Lane DP, Hall PA. p53 expression is common in malignant mesothelioma. Histopathology. 1992;21(4):331-4.

17. Metcalf RA, Welsh JA, Bennett WP, Seddon MB, Lehman TA, Pelin K, et al. P53 and Kirsten-ras mutations in human mesothelioma cell lines. Cancer Res. 1992;52(9):2610-5.

18. Cury PM, Butcher DN, Corrin B, Nicholson AG. The use of histological and immunohistochemical markers to distinguish pleural malignant mesothelioma and in situ mesothelioma from reactive mesothelial hyperplasia and reactive pleural fibrosis. $\mathrm{J}$ Pathol. 1999;189(2):251-7.

19. Roberts F, Harper CM, Downie I, Burnett RA. Immunohistochemical analysis still has a limited role in the diagnosis of malignant mesothelioma. A study of thirteen antibodies. Am J Clin Pathol. 2001;116(2):253-62.

20. Gundersen HJ, Bendtsen TF, Korbo L, Marcussen N, Moller A, Nielsen $\mathrm{K}$, et al. Some new, simple and efficient stereological methods and their use in pathological research and diagnosis. APMIS. 1988;96(5):379-94.

21. de Capitani EM, Metze K, Frazato Junior C, Altemani AM, Zambom L, Toro IF, et al. [Malignant mesothelioma of the pleura with etiological association to asbestos: report of 3 clinical cases] Rev Assoc Med Bras. 1997;43(3):265-72. Portuguese.

22. Pinheiro GA, Antão VC, Monteiro MM, Capelozzi VL, Terra-Filho M. Mortality from pleural mesothelioma in Rio de Janeiro, Brazil, 1979-2000: estimation from death certificates, hospital records and histopathological assessment. Int J Occup Environ Health. 2003;9(2):147-52. Comment in: Int J Occup Environ Health. 2003;9(2):171.

23. Hillerdal G. Mesothelioma: cases associated with non-occupational and low dose exposures. Occup Environ Med. 1999;56(8):505-13.

24. Enzinger FM, Weiss SW. Mesothelioma. In: Enzinger FM, Weiss SW. Soft tissue tumors. 2nd ed. St. Louis: Mosby; 1983. p. 689718.

25. Hasleton PS. Pleural disease. In: Hasleton PS. Spencer's pathology of the lung. 5th ed. New York: McGraw-Hill; 1996. p.1131-10.

26. Henderson DW, Comin CE, Hammar SP. Malignant mesothelioma of the pleura: current surgical pathology. In: Corrin B, editor. Pathology of lung tumors. New York: Churchill Livingstone; 1997. p.241-80.

27. Sheibani K, Battifora H, Burke JS. Antigenic phenotype of malignant mesotheliomas and pulmonary adenocarcinomas. An immunohistologic analysis demonstrating the value of Leu M1 antigen. Am J Pathol. 1986;123(2):212-9.

28. Wick MR, Loy T, Mills SE, Legier JF, Manivel JC. Malignant epithelioid pleural mesothelioma versus peripheral pulmonary adenocarcinoma: a histochemical, ultrastructural, and immunohistologic study of 103 cases. Hum Pathol. 1990;21(7):75966.

29. Comin CE, Novelli L, Boddi V, Paglierani M, Dini S. Calretinin, thrombomodulin, CEA, and CD15: a useful combination of immunohistochemical markers for differentiating pleural epithelial mesothelioma from peripheral pulmonary adenocarcinoma. Hum Pathol. 2001;32(5):529-36.

30. Gotzos V, Vogt P, Celio MR. The calcium binding protein calretinin is a selective marker for malignant pleural mesotheliomas of the epithelial type. Pathol Res Pract. 1996; 192(2):137-47. Erratum in: Pathol Res Pract. 1996;192(6): 646. Erratum in: Pathol Res Pract. 1996;192(6):646. 